\title{
ENHANCEMENT OF FUMARIC ACID PRODUCTION BY RHYZOPUS ORYZAE USING OXYGEN - VECTOR
}

\author{
CORINA CIOBANU ${ }^{a}$, ALEXANDRA TUCALIUC ${ }^{a, *}$, \\ ALEXANDRA CRISTINA BLAGA ${ }^{a}$, ANCA-IRINA GALACTION ${ }^{b}$, \\ DAN CAȘCAVAL ${ }^{a}$
}

\begin{abstract}
Fumaric acid, one of the most important organic acids with various applications in industries, can be obtained by fungal fermentation processes, mainly by using Rhizopus oryzae as producing microorganism. The challenges are to maintain pellet morphology of fungy and to optimize the multiple factors that influence fumaric acid production. The main objective of this study is to improve fumaric acid production by varying $n$-dodecane concentration levels in batch fungal fermentations. This strategy leads to an increase of fumaric acid concentration for about 1.2 times in system with $5 \%$ $n$-dodecane but a higher hydrocarbon concentration induced inhibition phenomenon to fumaric acid production.
\end{abstract}

Keywords: fumaric acid, fermentation, Rhizopus oryzae, oxygen-vector, ndodecane

\section{INTRODUCTION}

Fumaric acid (trans-butenedioic acid) is a four carbon dicarboxylic acid, one of the two isomeric unsaturated dicarboxylic acids, alongside maleic acid. Fumaric acid is found in certain species of fungus, lichen, and other plants, including Fumaria officinalis, from which it was first isolated [1].

It is a very important compound due to its numerous applications in food, feed and beverages, used as acidulant, preservative and flavoring agent; as intermediate for L-malic and L-aspartic acid production, and also as chemical feedstock for the production of paper resins, unsaturated polyester resins, alkyd resins, plasticizers, and miscellaneous industrial products [1, 2].

a "Gheorghe Asachi" Technical University of lasi, "Cristofor Simionescu" Faculty of Chemical Engineering and Environmental Protection, 73 Prof. Dimitrie Mangeron str., RO-700050, lasi, Romania

b "Gr.T. Popa" University of Medicine and Pharmacy of lasi, Dept. of Biomedical Science, 9-13 M. Kogalniceanu str., RO-700454, lasi, Romania

*Corresponding author: tucaliucalexandra@gmail.com 
The petrochemical routes for the fumaric acid production via catalytic isomerization of maleic acid or through maleic anhydride requires high material and energy consumptions, leading to the increased interest for fermentative processes. Some microorganisms, such as bacteria E. coli, Lactobacillus $s p$. produce fumaric acid in small amounts, as a key intermediate in the citrate cycle. Fumaric acid is produced especially by fungus; some identified genera are Rhizopus, Mucor, Cunninghamella, and Circinella. Among these strains, Rhizopus species (nigricans, arrhizus, oryzae, and formosa) were the bestproducing, yielding fumaric acid under aerobic and anaerobic conditions [3, 4].

Although high fumaric acid yields have been obtained from fungi such as Rhizopus oryzae and Rhizopus arrhizus, the process might be limited at the industrial scale because these fungi are difficult to grow and their morphology can strongly affect production characteristics [5, 6].

Fungi can be grown in submerged cultures in several different morphological forms: suspended mycelia, clumps, or pellets $[7,8]$. In terms of fumaric acid production, fungal growth in pellet form is a favorable alternative because significantly improves the rheology of the fermentation broth which results in efficient mass and oxygen transfer rates into the biomass and reduces energy consumption for aeration and agitation [9].

Rhizopus species are aerobic microorganisms that require a continuous supply of oxygen during the fermentation process. Variations in the dissolved oxygen (DO) level might have a significant effect on the production of fumaric acid and byproducts. Malic acid and ethanol are produced as byproducts, but in small concentrations. Ethanol production usually occurs under anaerobic conditions or when oxygen is limited in the culture, and thus can be decreased by providing sufficient oxygen to the culture [10].

Fumaric acid production depends on fungal cells growth and pellet formation in submerged cultures, process controlled by multiple factors such as nutrients, $\mathrm{pH}$, agitation, aeration, inoculum level, substrate concentration, polymer additives, the broth viscosity, and surface-active agents [11]. Among these factors, oxygen transfer rate (aeration) or the control of dissolved oxygen in the medium during fermentation is considered very important for organic acid production. In the case of fumaric acid, high concentrations of oxygen in the broth yield high titers of the acid, but low productivities, while at low $\mathrm{DO}$ the situation is reversed. A combination of high and low dissolved oxygen, in this order, permits high titers of fumaric acid and high glucose conversions [12].

An alternative of this strategy (combination of high and low dissolved oxygen) and without needing a supplementary intensification of mixing may be represented by the addition of some immiscible liquids in the aqueous phase which could induce a significant increase of oxygen transfer rate from air to microorganisms' cells. These compounds, in which oxygen is highly soluble, are known in literature as oxygen-vectors [13]. Some studies reporting the use of oxygen-vectors to improve the performance of fermentations processes [14, 15]. 
The aim of this study was to investigate the possibility to optimize the dissolved oxygen control for improving fumaric acid production by adding different levels of oxygen-vectors in fungal fermentation.

\section{RESULTS AND DISCUSSION}

Important parameters that require optimization in the fermentation process are medium composition and oxygen transfer rate (aeration) or the control of dissolved oxygen in the medium during fermentation. Factors affecting cell growth and pellet formation, including the strain, spore inoculum size, and culturing conditions (medium composition, temperature, $\mathrm{pH}$, and agitation speed) have been extensively studied [16].

For batch fermentations without oxygen-vector Figure 1 shows slow glucose consumption by the fungus pellets with a fast increase in fumaric acid accumulation in the broth after 100 hours, indicating that the carbon sources is consumed for pellets formation instead of acid biosynthesis.

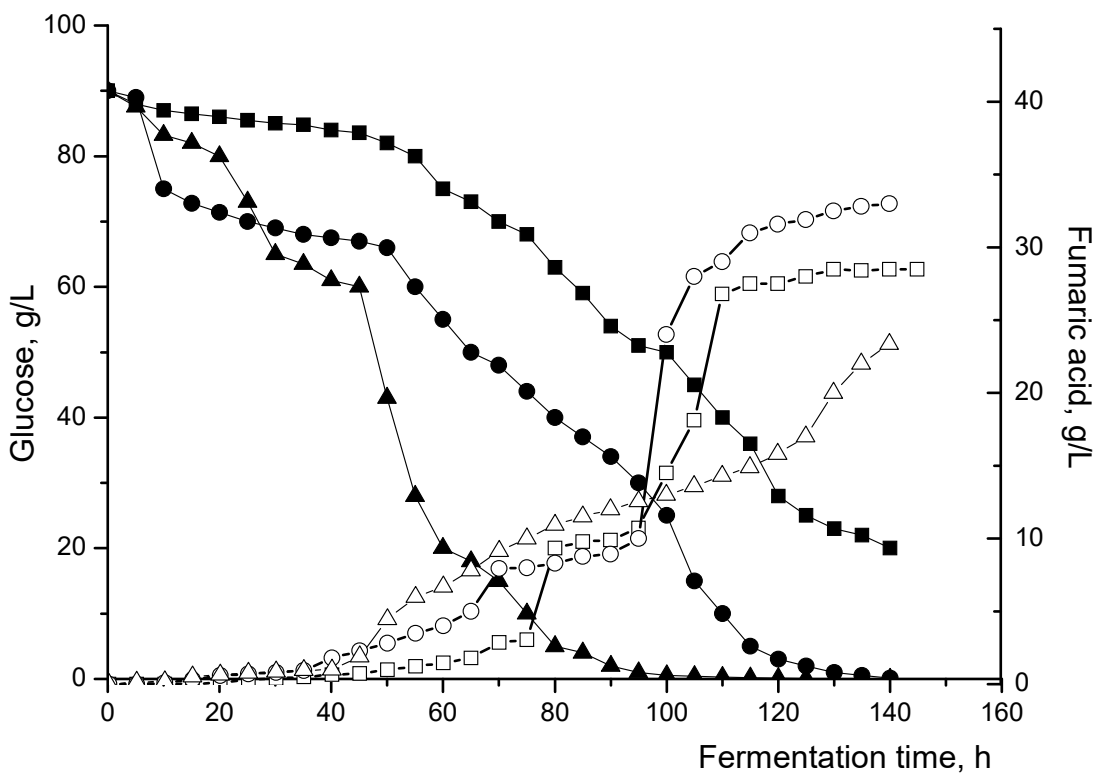

Figure 1. Variation of glucose and fumaric acid concentrations during fungal fermentation (glucose concentration: - $0 \% n$-dodecane, $\bullet-5 \% n$-dodecane,

$\Delta-10 \% n$-dodecane; fumaric acid concentration: $\square-0 \% n$-dodecane, $\circ-5 \% n$-dodecane, $\Delta-10 \% n$-dodecane) 
Hydrocarbon concentration exhibits a significant influence on fumaric acid production mainly by accelerating the oxygen transfer rate, therefore in the presence of $5 \% n$-dodecane the decrease of glucose consumption is more pronounced attaining the maximum final concentration of fumaric acid (33 $\left.\mathrm{g} \cdot \mathrm{L}^{-1}\right)$ in the broth. The concentration of fumaric acid remains at a constant level in absence of $n$-dodecane after $100 \mathrm{~h}$ of fermentation, but increases slowly in the presence of hydrocarbon. The oxygen vector addition improved glucose consumption: the substrate was completely exhausted for the fermentation with $n$-dodecane in $140 \mathrm{~h}$, while for the control system, the residual glucose was about $20 \mathrm{~g} \cdot \mathrm{L}^{-1}$. Therefore, due to a reduced concentration of dissolved oxigen in the absence of hydrocarbon, pellets could not use all the assimilable nitrogen, this leading to a slow fermentation and the process was stopped before the end of fermentable sugars.

For the system with $10 \% n$-dodecane, Figure 1 shows the significant decrease of glucose concentration during the first $60 \mathrm{~h}$, this substrate being almost entirely consumed over $80 \mathrm{~h}$. The $n$-dodecane addition leads to a superior rate of glucose consumption, due to an increased rate of pellets multiplication under higher dissolved oxygen concentration. This phenomenon is followed by exponential increasing of fumaric acid concentration, probably due to the consumption of $n$-dodecane as alternative source of carbon and energy, but at the end of fermentation process fumaric acid concentration in broth reached a low value $\left(23.35 \mathrm{~g}^{\cdot \mathrm{L}^{-1}}\right)$, as compared with systems without and with $5 \%$ hydrocarbon. This variation is a result of inhibition effect which appeared at higher hydrocarbon concentration. The increased values of DO determined by the high amount of oxygen vector in the medium were extremely beneficial for the biomass growth at the expense of acid production, since Rhizopus strains accumulate fumaric acid in stress conditions. If the medium offers optimum conditions (DO over $80 \%$ ) the carbon source will be used only for biomass accumulation, fungi being able to grow on hydrocarbon substrates $[17,18]$.

Fumaric acid production by fungal culture is limited by the dissolved oxygen in the broth, thus one way to minimize oxygen mass transfer limitation to the cells is to stimulate the formation of small, spherical, cell pellets, which can reduce clump formation and broth viscosity. During fungal fermentation of $R$. oryzae the process was towards pellets formation and pellets with a diameter over $1 \mathrm{~mm}$ were obtained (Figure 2.A.) in the absence of hydrocarbon, whereas in the presence of $n$-dodecane smaller pellets $(<1 \mathrm{~mm}$ diameter) (Figure 2.B. and Figure 2.C.) were formed. 

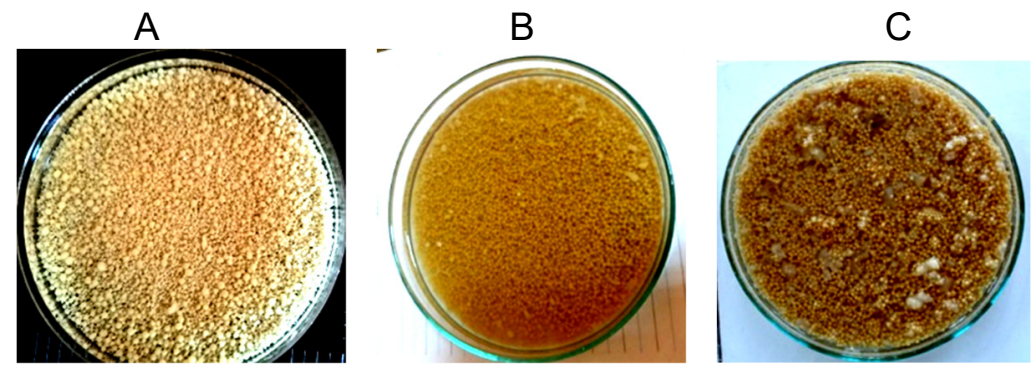

Figure 2. Close-up of the pellets formed in fermentation systems: A. without $n$-dodecane, B. with $5 \% n$-dodecane and C. with $10 \% n$-dodecane.

In the systems with smaller pellets more biomass was formed, leading to maximum biomass concentration of $5.5 \mathrm{~g} \cdot \mathrm{L}^{-1} \mathrm{~d}$.w. for system with $10 \%$ $n$-dodecane (Figure 3.), but it was not correlated with a higher content of fumaric acid. This fact indicates that a higher concentration of hydrocarbon $(10 \%$ $n$-dodecane) leads to biomass growth and inhibit the fumaric acid production.

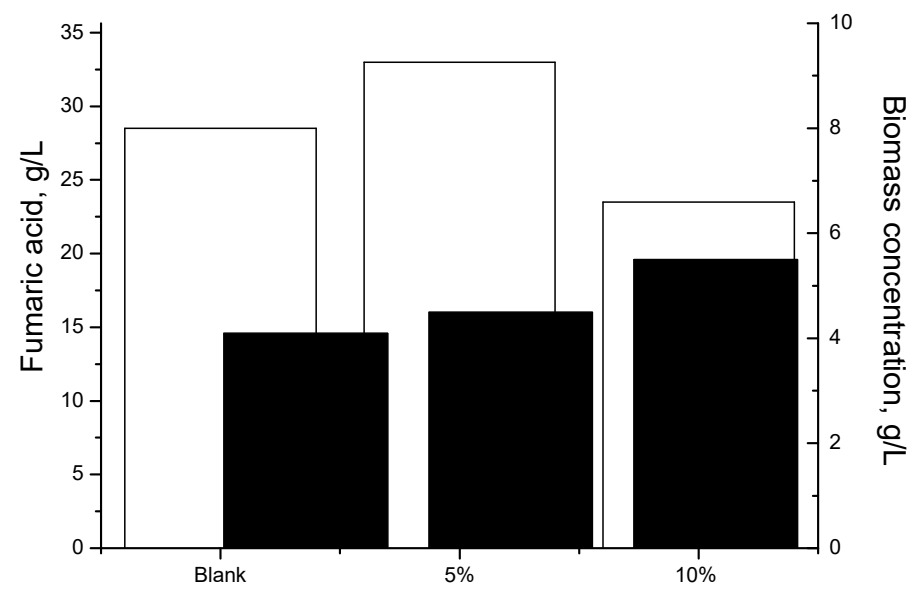

Figure 3. Correlation of fumaric acid production with biomass concentration ( $\square$ - fumaric acid concentration, - - biomass concentration).

Smaller pellets formation correlated with higher biomass concentration indicated an optimal oxygen mass transfer in the inner region of pellets due to the higher concentration of dissolved oxygen in the fermentation broth, generating a gradient concentration between pellets and culture medium. A more intensive metabolism of the smaller pellets and the presence of oxygen vector in the broth could prompt the dissolved oxygen, the most important substrate, to be easier and faster transferred into pellets. Decreasing the 
oxygen-vector concentration ( $5 \% n$-dodecane) the inhibition effect to fumaric acid production was eliminated, this leading to higher accumulation of fumaric acid $\left(33 \mathrm{~g} \cdot \mathrm{L}^{-1}\right)$ at the end of fermentation under limited pellets growth (biomass concentration $4.5 \mathrm{~g} \cdot \mathrm{L}^{-1} \mathrm{~d}$.w.).

Oxygen is the most difficult substrate to be supplied to aerobic cultures of $R$. oryzae pellets. According to Figure 4, the values of dissolved oxygen concentration are significantly higher in presence of hydrocarbon comparatively with control fermentation processes. This variation is due to the positive effect on the appearance of limiting steps, such as mass transfer of oxygen from bulk to boundary layer (the film surrounding the pellets) and to inner pellet diffusion step. For maintaining a constant level of oxygen in the pellets is important to provide a high level of oxygen in the fermentation broth, leading to a high concentration gradient between bulk and inner of the pellets. Therefore, the level of fumaric acid biosynthesised by pellets depends directly on substrate and oxygen concentration into the broth. After $80 \mathrm{~h}$ of fermentation the fumaric acid concentration increased continuously, but over 120 hours it reached a constant level in the system without $n$-dodecane, indicating that the conditions discussed above were not maintained and the substrate was consumed in a superficial region of the pellets, inducing the lyses of the cells in the inner of the pellets.

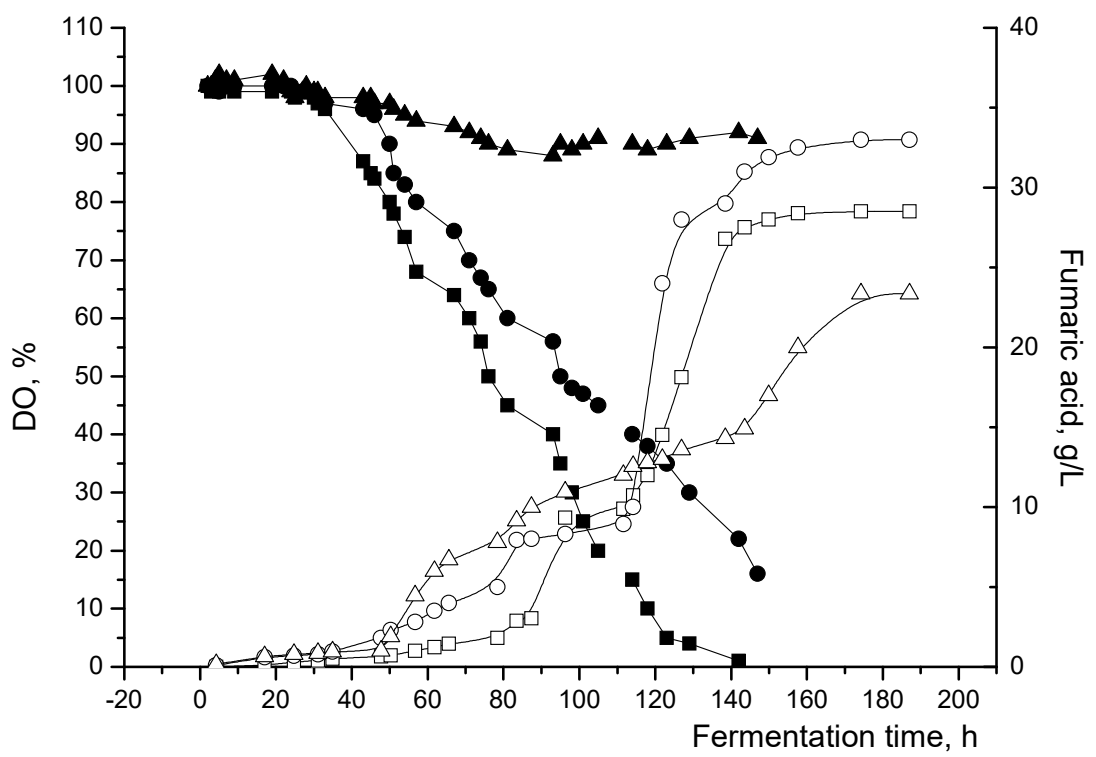

Figure 4. The profile of dissolved oxygen during fungal fermentation (dissolved oxygen concentration: - $0 \% n$-dodecane, $\bullet-5 \% n$-dodecane, $\boldsymbol{\Delta}$ - $10 \% n$-dodecane; fumaric acid concentration: $\square-0 \% n$-dodecane, $\circ-5 \% n$-dodecane, $\Delta-10 \% n$-dodecane). 
Whereas in the presence of $5 \% n$-dodecane, the pellets still produce fumaric acid after $120 \mathrm{~h}$ of fermentation, and reach a high concentration level in the broth as a result of the increased biomass amount and due to the positive influence of hydrocarbon on the oxygen mass transfer in the inner region of the pellets. Regarding the system with $10 \% n$-dodecane, the dissolved oxygen is maintained at relatively high values during the entire fermentation processes (Figure 4) and limits the fumaric acid biosynthesis rate due to the inhibitory effect on the pellets production step.

Oxygen mass transfer proprieties are very important in the complex media of fungal fermentation, this representing an important key for process scale-up and commercialisation. Therefore, the influence of $n$-dodecane concentration on oxygen transfer rate, via oxygen transfer coefficient $\left(k\left\llcorner a, s^{-1}\right)\right.$, was analysed. According to Figure 5 the positive influence of hydrocarbon is correlated by the amplification of the oxygen mass transfer coefficient for about 1.3 times by increasing the volumetric concentration of dodecane from 0 to $5 \%$.

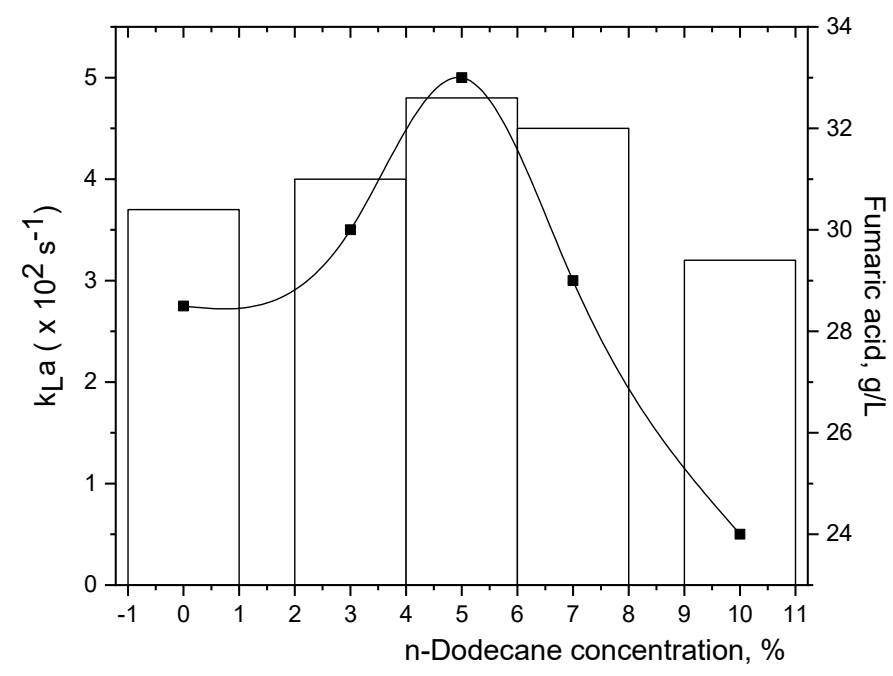

Figure 5. Influence of $n$-dodecane concentration on oxygen mass transfer coefficient and fumaric acid concentration.

As mentioned above, higher hydrocarbon concentration led to the appearance of the inhibition effect for fumaric acid production, which is confirmed by the decrease of the k $\mathrm{L}$ a coefficient for about 1.5 times in systems with $10 \%$ n-dodecane. Therefore, higher concentration of oxygen-vectors limits the production of fumaric acid, but this negative influence is partially countered by pellets accumulation in the fermentation broth. 


\section{CONCLUSIONS}

Fumaric acid production by fungal fermentation is strongly dependent on dissolved oxygen concentration, therefore a strategy to improve the biosynthesis performance was proposed. The experiments were carried out in batch systems and fungus morphology was controlled for pellets formation. Batch fermentations were performed in the absence and presence of $n$-dodecane, used as oxygen vector. Hydrocarbon concentration exhibits a significant influence on fumaric acid production. Therefore, in the presence of $5 \% n$-dodecane, the decrease of glucose consumption is more pronounced, leading to an increase of fumaric acid concentration of about 1.2 times. At higher hydrocarbon concentration (10\% $n$-dodecane) an inhibition effect on fumaric acid production appeared which can be assumed to the cumulative negative effect of two factors: attaining the inhibition level of hydrocarbon and the high amounts of biomass in the fermentation broth. The maximum amount of fumaric acid was reached with $5 \%$ n-dodecane, while in systems with $10 \% n$-dodecane the fumaric acid production decreased with almost $50 \%$.

\section{EXPERIMENTAL SECTION}

The fungal batch fermentations were performed in a laboratory stirred bioreactor (2 L Fermentor Fermac 360, Electrolab, UK) with 1.4 L. working volume. The characteristics of bioreactor are given in Table 1.

Table 1. Geometric characteristics of bioreactor $(\mathrm{d}=$ impeller diameter, $\mathrm{mm}$; $\mathrm{D}=$ bioreactor diameter, $\mathrm{mm} ; \mathrm{h}=$ distance from the stirrer to the bioreactor bottom, $\mathrm{mm}$;

$\mathrm{H}=$ bioreactor height, $\mathrm{mm} ; \mathrm{I}=$ impeller blade length, $\mathrm{mm}$; l' = oxygen electrode immersed length, $\mathrm{mm} ; \mathrm{w}=$ impeller blade height, $\mathrm{mm}$ )

\begin{tabular}{|c|c|c|c|c|c|c|c|c|}
\hline $\mathbf{d}$ & $\mathbf{d} / \mathbf{D}$ & H/D & w/d & l/d & h/d & d'/d & l'/d & $\begin{array}{c}\text { no. } \\
\text { blades }\end{array}$ \\
\hline 55 & 0.46 & 1.46 & 0.27 & 0.31 & 0.64 & 0.018 & 1.82 & 6 \\
\hline
\end{tabular}

As producing microorganism, Rhizopus oryzae ATCC 20344 was used. $R$. oryzae was cultured on malt extract agar at $35^{\circ} \mathrm{C}$ for 7 days. The agar with fungi spores was washed with sterile water. The spore suspensions were used to inoculate the pre-culture medium. The composition of the growth medium for spores was: glucose $30 \mathrm{~g} \cdot \mathrm{L}^{-1}$, yeast extract $2.5 \mathrm{~g} \cdot \mathrm{L}^{-1}$, $\mathrm{KH}_{2} \mathrm{PO}_{4} 0.6 \mathrm{~g} \cdot \mathrm{L}^{-1}, \mathrm{MgSO}_{4} 0.25 \mathrm{~g} \cdot \mathrm{L}^{-1}, \mathrm{ZnSO}_{4} 0.044 \mathrm{~g} \cdot \mathrm{L}^{-1}, \mathrm{FeCl}_{3} 0.0075 \mathrm{~g} \cdot \mathrm{L}^{-1}$. 
The medium without the glucose was heat sterilized (20 minute at $121^{\circ} \mathrm{C}$ ), the glucose was sterilized separately. Precultures were carried out at $35^{\circ} \mathrm{C}$ and $180 \mathrm{rpm}$ in a gyratory incubator-shaker for 1-2 days. After cultivation, the obtained pellets were transferred immediately into a fermenter containing the production medium: glucose $90 \mathrm{~g} \cdot \mathrm{L}^{-1},\left(\mathrm{NH}_{4}\right)_{2} \mathrm{SO}_{4} 2 \mathrm{~g} \cdot \mathrm{L}^{-1}, \mathrm{KH}_{2} \mathrm{PO}_{4} 0.3 \mathrm{~g} \cdot \mathrm{L}^{-1}$, $\mathrm{MgSO}_{4} 0.25 \mathrm{~g} \cdot \mathrm{L}^{-1}, \mathrm{ZnSO}_{4} 0.044 \mathrm{~g} \cdot \mathrm{L}^{-1}, \mathrm{FeCl}_{3} 0.0075 \mathrm{~g} \cdot \mathrm{L}^{-1} . n$-Dodecane was used as oxygen vector at volumetric concentration of $5 \%$ and $10 \%$. For the $\mathrm{pH}$ control a saturated solution of $\mathrm{Na}_{2} \mathrm{CO}_{3}$ sterilized by filtration was used. Silicone-based antifoam agent for controlling foam generation during batch fermentations was used.

Fermentation processes were carried out in the following conditions: $35{ }^{\circ} \mathrm{C}, 400 \mathrm{rpm}, \mathrm{pH} 5.5$, aeration rate $2 \mathrm{~L} \cdot \mathrm{min}^{-1}$, fermentation time approx. $160-180$ hours. Samples were taken periodically and the glucose and fumaric acid contents were monitored using a HPLC system (Dionex Ultimate 3000). Glucose analysis was performed on a HPLC system equipped with refractive index detector using HyperRez carbohydrate column $(300 \times 7.7 \mathrm{~mm}$, $8 \mu \mathrm{m}$ ) with water as mobile phase at $0.6 \mathrm{~mL} \cdot \mathrm{min}^{-1}$ and column temperature of $80^{\circ} \mathrm{C}$, while the fumaric acid was analyzed by a PDA detector and Acclaim OA column $(150 \times 4.5 \mathrm{~mm}, 5 \mu \mathrm{m})$. The mobile phase consisted in $100 \mathrm{mM}$ $\mathrm{Na}_{2} \mathrm{SO}_{4}$ solution, $\mathrm{pH} 3.65$ corrected with methane sulfonic acid, at a flow rate of $0.6 \mathrm{~mL} \cdot \mathrm{min}^{-1}$ and $30^{\circ} \mathrm{C}$.

\section{ACKNOWLEDGMENTS}

This work was supported by a research grant of the TUIASI, project number 0973/2018.

\section{REFERENCES}

1. A.C. Roa Engel, A.J.J. Straathof, T.W. Zijlmans, W.M. van Gulik, L.A.M. van der Wielen, Appl. Microbiol. Biotechnol., 2008, 78, 379-389.

2. Q. Xu, S. Li, H. Huang, J. Wen, Biotechnol. Adv., 2012, 30, 1685-1696.

3. E. Riscaldati, M. Moresi, F. Federici, M. Petruccioli, Biotechnol. Lett., 2000, 22, 1043-1047.

4. E. Podgórska, M. Kasprzak, D. Szwajgier, Pol. J. Food Nutr. Sci., 2004, 54, 47-50.

5. Y.Q. Fu, Q. Xu, S. Li, Y. Chen, H. Huang, Korean J. Chem. Eng., 2010, 27, 183186.

6. W. Kenealy, E. Zaady, J.C. Dupreez, B. Stieglitz, I. Goldberg, Appl. Environ. Microbiol., 1986, 52, 128-133. 
CORINA CIOBANU, ALEXANDRA TUCALIUC, ALEXANDRA CRISTINA BLAGA,

ANCA-IRINA GALACTION, DAN CAȘCAVAL

7. B. Metz, N.W.F. Kossen, Biotechnol. Bioeng., 1977, 19, 781-799.

8. W. Liao, Y. Liu, S. Chen, Appl. Biochem. Biotechnol., 2007, 137, 689-701.

9. J.C. van Suijdam, N.W.F. Kossen, P.G. Paul, Eur. J. Appl. Microbiol. Biotechnol., 1980, 10, 211-221.

10. N. Cao, J. Du, C.S. Gong, G.T. Tsao, Appl. Environ. Microbiol., 1996, 62, 2926-31.

11. J. Zhang, J. Zhang, Crit. Rev. Biotechnol., 2016, 36, 1066-1077.

12. Y.Q. Fu, S. Li, Y. Chen, Q. Xu, H. Huang, X. Y. Sheng, Biotechnol. Appl. Biochem., 2010, 162, 1031-1038.

13. J.L. Rols, J.S. Condoret, C. Fonade, G. Goma, Biotechnol. Bioeng.,1990, 35, 427-435.

14. S. Jia, M. Wang, P. Kahar, Y. Park, M. Okabe, J. Ferment. Bioeng., 1997, 84, 176-178.

15. A.C. Blaga, C. Ciobanu, D. Cascaval, A.-I. Galaction, Biochem. Eng. J., 2018, $131,70-76$.

16. M. Papagianni, Biotechnol. Adv., 2004, 22, 189-259.

17. F.X. Prenafeta-Boldú, A. Kuhn, D.M.A. M. Luykx, H. Anke, J.W. van Groenestijn, J.A.M. de Bont, Mycol. Res., 2001, 105, 477-484.

18. F.X. Prenafeta-Boldú, R. Summerbell, G. Sybren de Hoog, FEMS Microbiol. Rev., 2006, 30, 109-130. 